\title{
Nematoides fitoparasitos em áreas de cultivo de antúrio dos estados de São Paulo, Paraná e Santa Catarina
}

\author{
Plant parasitic nematodes in anthurium growing areas from \\ São Paulo, Paraná and Santa Catarina States, Brazil
}

\section{Claudio Marcelo Gonçalves de Oliveira ${ }^{\mathrm{I}^{*}}$ Rosana Bessi $^{\mathrm{II}}$ Juliana Magrinelli Osório Rosa Roberto Kazuhiro Kubo' Antonio Fernando Caetano Tombolato III}

\section{- NOTA -}

RESUMO

$O$ objetivo do presente trabalho foi identificar os nematoides associados a plantas de antúrio (Anthurium andraeanum) cultivadas como flor de corte (13 amostras) ou plantas em vaso (41 amostras) nos estados de São Paulo, Paraná e Santa Catarina. Nematoides foram extraídos de $10 \mathrm{~g}$ de raizes e $250 \mathrm{~cm}^{3}$ de solo ou substrato e identificados sob microscópio de luz. A espécie mais frequente foi Radopholus similis, detectada em $54 \%$ das amostras de antúrio cultivado como flor de corte. Constituíram novas ocorrências em antúrio no Brasil: Helicotylenchus erythrinae, $\boldsymbol{H}$. californicus, $\boldsymbol{H}$. multicinctus, Meloidogyne paranaensis e $\boldsymbol{R}$. similis. Entretanto, nenhum nematoide parasito de plantas foi encontrado nos antúrios cultivados em vaso, com substrato de fibra de coco.

Palavras-chave: Anthurium andraeanum, levantamento, primeira ocorrência.

\section{ABSTRACT}

The aim of this research was to identify plant parasitic nematodes associated with Anthurium andraeanum plants cultivated as cut flowers (13 samples) or pot flowers (41 samples) in the States of São Paulo, Paraná and Santa Catarina, Brazil. Nematodes were extracted from $10 \mathrm{~g}$ of roots and $250 \mathrm{~cm}^{3}$ of soil by centrifugation method and identified under microscope. The most frequently occurring species was Radopholus similis detected on 54\% of anthuriums cultivated as cut flowers. Among the identified species, the following associations constitute new records to Brazil in anthuriums: Helicotylenchus erythrinae, $\boldsymbol{H}$. californicus, $\boldsymbol{H}$. multicinctus, Meloidogyne paranaensis and $\boldsymbol{R}$. similis. However, no plant parasitic nematodes were detected on $\boldsymbol{A}$. andraeanum cultivated as pot flowers in coconut fiber substrate.

Key words: Anthurium andraeanum, first record, survey
O estado de São Paulo é considerado um dos maiores centros de produção e comercialização do antúrio (Anthurium andraeanum Linden), cuja produção se concentra nas regiões de Holambra, Atibaia e Vale do Ribeira (CALDARI JÚNIOR, 2004). No Brasil, mais de 20 gêneros de fitonematoides foram detectados em associação a plantas ornamentais (OLIVEIRA, 2008). Afora relatos isolados de novas ocorrências, a literatura brasileira registra apenas seis levantamentos de nematoides em plantas ornamentais, realizados em Lavras, Minas Gerais (COSTA et al., 2001), no norte e noroeste do Paraná (DIAS-ARIEIRA et al., 2007), na zona da mata de Pernambuco (LINS \& COELHO, 2004), nos estados de São Paulo e Minas Gerais (OLIVEIRA et al., 2007), no estado do Ceará (FREIRE \& MOSCA, 2009) e no litoral sul da Bahia (MATTOS SOBRINHO et al., 2012). Portanto, ainda não há referência de nematoides associados a um grande número de espécies de plantas ornamentais, incluindo-se nesse caso, o antúrio.

Atualmente, no Brasil, são conhecidas as associações das seguintes espécies de nematoides ao antúrio: Helicotylenchus dihystera (Cobb) Sher, Meloidogyne incognita (Kofoid \& White) Chitwood, M. javanica (Treub) Chitwood, M. inornata Lordello, Scutellonema brachyurus (Steiner) Andrassy, Xiphinema sp. Cobb e Tylenchus sp. Bastian (COSTA MANSO et al., 1994; COSTA et al., 2001; DIASARIEIRA et al., 2007; OLIVEIRA, 2008; FREIRE

\footnotetext{
ILaboratório de Nematologia, Instituto Biológico, 13001-970, Campinas, SP, Brasil. E-mail: marcelo@biologico.sp.gov.br. *Autor para correspondência.

"Escola Superior de Agricultura "Luiz de Queiroz" (ESALQ), Piracicaba, SP, Brasil.

IIINúcleo de Pesquisa e Desenvolvimento do Jardim Botânico, Instituto Agronômico de Campinas (IAC), Campinas, SP, Brasil. Recebido 11.01.13 Aprovado 18.09.14 Devolvido pelo autor 16.03.15 CR-2013-0040.R2
} 
\& MOSCA, 2009). Apesar de existirem os relatos citados, a avaliação apropriada dos danos causados por essas espécies de nematoides em antúrio ainda não foi realizada. Assim, o presente trabalho teve como objetivos estudar a ocorrência de nematoides fitoparasitos nas áreas de cultivo de antúrio do estado de São Paulo, Paraná e Santa Catarina.

Treze amostras nematológicas compostas de solo e de raízes foram coletadas nas principais áreas produtoras de antúrio, cultivados como flor de corte nos municípios de Pariquera-Açu (SP), Caraguatatuba (SP), Garuva (PR) e Joinvile (SC). Cada subamostra foi formada por aproximadamente $10 \mathrm{~g}$ de raízes jovens e $250 \mathrm{~cm}^{3}$ de solo da rizosfera de antúrio, coletadas à profundidade de 0 a $20 \mathrm{~cm}$. Cada amostra foi constituída de quatro subamostras, coletadas aleatoriamente em diferentes pontos de cada canteiro, sendo devidamente acondicionados em sacos de polietileno, etiquetados e armazenados em caixas térmicas para transporte ao laboratório.

Além disso, também estão registradas, no presente trabalho, 41 amostras de substrato (fibra de coco) e raízes de antúrio cultivados em vaso, provenientes dos municípios paulistas de Artur Nogueira, Campinas, Jaguariúna, Holambra e Santo Antônio de Posse, as quais foram encaminhadas para análise nematológica à clínica mantida pelo laboratório de Nematologia do Centro Experimental Central do Instituto Biológico (CEIB/Instituto Biológico), em Campinas (SP), no período de 2010 a 2012.

Os nematoides presentes em $250 \mathrm{~cm}^{3}$ de solo ou substrato e $10 \mathrm{~g}$ de raízes foram extraídos pelos métodos de JENKINS (1964) e COOLEN \& D'HERDE (1972), respectivamente, sendo os espécimes mortos em água aquecida (banho-maria) a $60 \mathrm{C}$ por 5 minutos, fixados e conservados em formalina $2 \%$. A estimativa populacional de cada gênero foi obtida através da contagem em lâminas de Peters, utilizando-se microscópio de luz. Para a identificação das espécies, recorreu-se a lâminas temporárias (formalina) e/ou permanentes (glicerina), examinadas em microscópio provido de câmara clara. A identificação foi feita principalmente a partir do exame dessas lâminas, baseada em características morfológicas e morfométricas. A identificação de Meloidogyne Goeldi foi realizada através da análise da configuração perineal de fêmeas adultas e confirmada através de técnicas eletroforéticas do perfil de esterases (OLIVEIRA et al., 2012).

Nenhuma das 41 amostras de substrato e raízes de antúrio cultivados em vaso apresentou nematoides parasitos de plantas, provavelmente reflexo do uso de substrato (fibra de coco) livre de nematoides. Por outro lado, a partir de amostras de solo e de raízes obtidas de antúrios cultivados como flor de corte, diretamente no solo, em canteiros, detectou-se a presença de nematoides pertencentes aos gêneros Helicotylenchus Steiner, Meloidogyne, Paratylenchus Micoletzky e Radopholus Thorne. As espécies H. erythrinae (Zimmermann) Golden, $\boldsymbol{H}$. californicus Sher, $\boldsymbol{H}$. multicinctus (Cobb) Golden, e $\boldsymbol{R}$. similis (Cobb) Thorne, as quais foram identificadas com base em caracteres morfológicos e morfométricos inerentes a cada espécie, enquanto que $M$. paranaensis foi identificada pelo padrão perineal e perfil de esterase. As espécies identificadas constituem novas ocorrências em antúrio no Brasil.

Os dados quantitativos dos nematoides encontrados em 10 gramas de raiz e $250 \mathrm{~cm}^{3}$ de solo estão na tabela 1. Das 13 amostras oriundas dos municípios de Pariquera-Açu, Caraguatatuba, Garuva e Joinvile, a espécie mais frequente em raízes de antúrio foi $\boldsymbol{R}$. similis (presente em 54\% das amostras), seguido de Paratylenchus sp. (31\%) e M. paranaensis (15\%). Nas análises de solo, detectou-se $\boldsymbol{H}$. erythrinae em $23 \%$ das amostras, $\boldsymbol{H}$. multicinctus em 15\%, Paratylenchus sp. em 15\% e H. californicus em $8 \%$, sendo que $39 \%$ das amostras de solo não apresentavam fitonematoides.

Nas amostras de solo coletadas na rizosfera de antúrio 'IAC Isla', 'IAC Netuno' e 'IAC Juréia', em Caraguatatuba (SP), foi possível detectar três espécies de Helicotylenchus (H. erythrinae, $\boldsymbol{H}$. californicus e $\boldsymbol{H}$. multicinctus), ocorrendo concomitantemente a $\boldsymbol{R}$. similis. Helicotylenchus multicinctus é importante parasito de bananeira (GOWEN et al., 2005), tendo sido relatado também em Heliconia sp. L. e Musa ornata Roxb nos municípios de Conceição do Almeida e Cruz das Almas (BA) (ZEM \& LORDELLO, 1983).

Helicotylenchus erythrinae, juntamente com $\boldsymbol{H}$. crenacauda e $\boldsymbol{H}$. dihystera, foram relatadas pela primeira vez em Heliconia spp. no litoral sul da Bahia (MATTOS SOBRINHO et al., 2012). Helicotylenchus erythrinae está associada a várias plantas cultivadas no Brasil (COSTA MANSO et al., 1994), ocorrendo naturalmente em florestas de Mata Atlântica e em áreas de preservação permanente (SILVA et al., 2008). No entanto, apesar da ampla gama de plantas hospedeiras, até o presente não há relatos de danos de $\boldsymbol{H}$. erythrinae em antúrio ou outras plantas cultivadas. Com relação a $\boldsymbol{H}$. californicus, encontrado na rizosfera de antúrio 'IAC Netuno' cultivado em Caraguatatuba (SP), há relatos anteriores dessa espécie em diversas frutíferas (banana, laranja, mamão), além de coco e cana de 
Tabela 1 - Número de nematoides fitoparasitos associados ao antúrio, extraídos de 10 gramas de raiz e $250 \mathrm{~cm}^{3}$ de solo.

\begin{tabular}{|c|c|c|c|c|}
\hline Espécie & Cultivar de antúrio & Raiz & Solo & Procedência \\
\hline \multirow{3}{*}{ Helicotylenchus erythrinae } & 'IAC Isla' & 0 & 310 & Caraguatatuba (SP) \\
\hline & 'IAC Netuno' & 0 & 120 & Caraguatatuba (SP) \\
\hline & ‘IAC Juréia’ & 0 & 40 & Caraguatatuba (SP) \\
\hline \multirow{2}{*}{ Helicotylenchus multicinctus } & 'IAC Isla' & 20 & 100 & Caraguatatuba (SP) \\
\hline & 'IAC Juréia' & 10 & 10 & Caraguatatuba (SP) \\
\hline Helicotylenchus californicus & 'IAC Netuno' & 0 & 20 & Caraguatatuba (SP) \\
\hline \multirow{7}{*}{ Radopholus similis } & 'IAC Juréia' & 210 & 10 & Pariquera-Açu (SP) \\
\hline & 'IAC Iguape' & 110 & 10 & Pariquera-Açu (SP) \\
\hline & 'IAC Isla' & 30 & 10 & Pariquera-Açu (SP) \\
\hline & 'Anthura Champion' & 620 & 10 & Pariquera-Açu (SP) \\
\hline & 'IAC Isla' & 620 & 10 & Caraguatatuba (SP) \\
\hline & 'IAC Netuno' & 363 & 10 & Caraguatatuba (SP) \\
\hline & 'IAC Juréia’ & 230 & 10 & Caraguatatuba (SP) \\
\hline \multirow{2}{*}{ Meloidogyne paranaensis } & 'IAC Eidibel' & 1000 & 50 & Pariquera-Açu (SP) \\
\hline & 'IAC Astral' & 18000 & 120 & Pariquera-Açu (SP) \\
\hline \multirow{4}{*}{ Paratylenchus sp. } & 'IAC Eidibel' & 120 & 0 & Garuva (PR) \\
\hline & 'IAC Luau’ & 60 & 0 & Garuva (PR) \\
\hline & 'IAC Eidibel' & 230 & 140 & Joinvile (SC) \\
\hline & ‘IAC Juréia’ & 10 & 10 & Joinvile (SC) \\
\hline
\end{tabular}

açúcar, cultivados principalmente nos estados da Bahia e Sergipe (COSTA MANSO et al., 1994).

O gênero Paratylenchus foi detectado em amostras de solo e associados a raízes de antúrio provenientes de Garuva (PR) e Joinvile (SC). Os nematoides deste gênero são ectoparasitos, de ocorrência relativamente comum em solos brasileiros, com ampla gama de plantas hospedeiras, como, por exemplo, abacaxi, banana e coco (COSTA MANSO et al., 1994).

No presente trabalho, através da análise dos fenótipos de esterase (fenótipo P1) e padrão da região perineal de fêmeas, diagnosticou-se $\boldsymbol{M}$. paranaensis em antúrio 'IAC Eidibel' e 'IAC Astral' cultivados no município de Pariquera-Açu (SP). No geral, as plantas mostravam pequenas galhas e massas de ovos no sistema radicular. Anteriormente, exemplares de M. incognita haviam sido relatados nessa ornamental nos estados do Paraná (SANTOS \& SILVA, 1984) e Ceará (FREIRE \& MOSCA, 2009) e exemplares de M. inornata em São Paulo (LORDELLO, 1957). Nesse caso, as galhas encontradas eram semelhantes às relatadas no presente estudo. Além da ocorrência de M. incognita em áreas de cultivo de antúrio no Ceará, FREIRE \& MOSCA (2009) evidenciam o uso de solos contaminados na preparação dos substratos pelos produtores da região, que, em sua maioria, apresentam baixo nível tecnológico, resultando na disseminação desse e de outros patógenos associados às plantas ornamentais.

Radopholus similis, espécie migradora e endoparasita de muitas plantas tropicais, ocorre com frequência em raízes e rizomas de $\boldsymbol{A}$. andraeanum, causando a severa doença denominada de declínio do antúrio, responsável por perdas de $50 \%$ de produtividade (ARAGAKI et al., 1984). A. andraeanum é muito suscetível ao ataque de $\boldsymbol{R}$. similis, sendo que o nematoide cavernícola é considerado importante parasito dessa ornamental nos EUA (Flórida e Havaí), Trindade e Tobago e Portugal (Ilha da Madeira) (VOVLAS et al., 2003; BALA \& HOSEIN, 1996; SIPES \& LICHTY, 2002). Apesar da presença frequente em áreas brasileiras cultivadas com bananeira, às vezes próximas ao cultivo de antúrio, até o momento não existem registros de ocorrência do nematoide cavernícola em antúrios no Brasil. No presente estudo, $R$. similis foi encontrado parasitando o sistema radicular das cultivares 'IAC Juréia', 'IAC Iguape', 'IAC Isla', 'Anthura Champion' e 'IAC Netuno', provenientes de Pariquera-Açu (SP) e Caraguatatuba (SP). As raízes apresentavam lesões necróticas características do ataque dessa espécie, mas não foram observados sintomas reflexos na parte aérea. 
Através do presente trabalho, concluiu-se que antúrios cultivados em vasos, em substrato de fibra de coco, não apresentaram nematoides parasitos de plantas. No entanto, identificou-se, pela primeira vez no Brasil, associadas às plantas de antúrio destinadas a flor de corte, as espécies $\boldsymbol{H}$. erythrinae, H. californicus, H. multicinctus, M. paranaensis e $\boldsymbol{R}$. similis. Com exceção de $\boldsymbol{R}$. similis, não se conhece a extensão dos danos causados por esses nematoides, sendo necessários futuros estudos de patogenicidade.

\section{REFERÊNCIAS}

ARAGAKI, M. et al. Nature and control of Anthurium decline. Plant Disease, v.68, p.509-511, 1984. Disponível em: $<$ http://www.apsnet.org/publications/PlantDisease/BackIssues/ Documents/1984Articles/PlantDisease68n06_509.pdf>. Acesso em: 03 mar. 2012.

BALA, G.; HOSEIN, F. Plant-parasitic nematodes associated with Anthuriums and other tropical ornamentals. Nematropica, v.26, p.9-14, 1996. Disponível em: <http://journals.fcla.edu/ nematropica/article/view/64141/61809>. Acesso em: 18 nov. 2012.

CALDARI JÚNIOR, P. Técnicas de cultivo de antúrio. Revista Brasileira de Horticultura Ornamental, v.10, p.43-45, 2004. Disponível em: <http://www.sbfpo.com.br/rbho/index.php/rbho/ article/viewFile/325/318>. Acesso em: 03 mar. 2012.

COOLEN, W.A.; D'HERDE, C.J. A method for the quantitative extraction of nematodes from plant tissue. Merelbeke: State Nematology and Entomology Research Station, 1972. 77p.

COSTA MANSO, E. et al. Catálogo de nematoides fitoparasitos encontrados associados a diferentes tipos de plantas no Brasil. Brasília: Empresa Brasileira de Pesquisa AgropecuáriaEMBRAPA, 1994. 488p. (Catálago Técnico).

COSTA, M.J.N. et al. Nematóides em plantas ornamentais. Ciência e Agrotecnologia, v.25, p.1127-1132, 2001. Disponível em: $<$ http://www.editora.ufla.br/_adm/upload/revista/25-5-2001_11. pdf>. Acesso em: 10 set. $201 \overline{2}$.

DIAS-ARIEIRA, C.R. et al. Nematóides associados a plantas ornamentais em viveiros do Paraná. Nematologia Brasileira, v.31, p.46-55, 2007. Disponível em: <http://docentes.esalq.usp.br/sbn/ nbonline/ol\%20311/46-53\%20co.pdf>. Acesso em: 03 mar. 2012.

FREIRE, F.C.O.; MOSCA, J.L. Patógenos associados a doenças de plantas ornamentais no Estado do Ceará. Revista Brasileira de Horticultura Ornamental, v.15, p.83-89, 2009. Disponível em: <http://www.alice.cnptia.embrapa.br/bitstream/doc/631390/1/ PC09009.pdf>. Acesso em: 07 dez. 2012.

GOWEN, S.R. et al. Nematode parasites of bananas and plantains. In: LUC, M. et al. Plant parasitic nematodes of subtropical and tropical agriculture. King's Lynn, UK: CABI Publishing, 2005. p.611-643.
JENKINS, W.R. A rapid centrifugal flotation technique for separating nematodes from soil. Plant Disease Reporter, v.48, p.692, 1964

LINS, S.R.O.; COELHO, R.S.B. Ocorrência de doenças em plantas ornamentais tropicais no estado de Pernambuco. Fitopatologia Brasileira, v.29, p.332-335, 2004. Disponível em: <http://dx.doi. org/10.1590/S0100-41582004000300019>. Acesso em: 23 out. 2012. doi: $10.1590 / \mathrm{S} 0100-41582004000300019$.

LORDELLO, L.G.E. A note on nematode parasites of red anthurium (Anthurium andraeanum Lind.), with a description of Rotylenchus boocki. Nematologica, v.2, p.273-276, 1957. Disponível em: <http://dx.doi.org/10.1163/187529257X00338>. Acesso em: 14 abr. 2012. doi: 10.1163/187529257X00338.

MATTOS SOBRINHO, C.C. et al. Fitonematoides associados à Heliconia spp. em cultivos comerciais no litoral Sul da Bahia, Brasil. Nematropica, v.42, p.349-353, 2012.

OLIVEIRA, C.M.G. Nematoides parasitos de plantas ornamentais. In: ALEXANDRE, M.A.V. Plantas ornamentais: doenças e pragas. São Paulo: Instituto Biológico, 2008. p.189-206.

OLIVEIRA, C.M.G. et al. Nematoides. In: EIRAS, M.; GALETTI, S.R. Técnicas de diagnóstico de fitopatógenos. São Paulo: Devir. 2012. p.101-135.

OLIVEIRA, C.M.G. et al. Ocorrência de nematóides fitoparasitos em plantas ornamentais nos estados de São Paulo e Minas Gerais. Revista Brasileira de Horticultura Ornamental, v.13, p.135141, 2007. Disponível em: <http://www.sbfpo.com.br/rbho/index. php/rbho/article/viewFile/217/113>. Acesso em: 03 mar. 2012.

SANTOS, B.B.; SILVA, L.A.T. Ocorrência de nematóides do gênero Meloidogyne Goeldi 1887 (Nemata: Heteroderidae) em algumas plantas cultivadas do Estado do Paraná, Brasil. Revista Agricultura, v.59, p.21-26, 1984.

SILVA, R.A. et al. Fauna de fitonematóides na Mata Atlântica do vale do Ribeira, estado de São Paulo, Brasil. Nematropica, v.38, p.1-12, 2008. Disponível em: <http://journals.fcla.edu/ nematropica/article/view/64443/62111>. Acesso em: 24 set. 2012.

SIPES, B.S.; LICHTY, J.S. Radopholus similis damage to Anthurium andraeanum. Nematropica, v.32, p.77-81, 2002. Disponível em: <http://journals.fcla.edu/nematropica/article/ view/69646/67306>. Acesso em: 03 mar. 2012.

VOVLAS, N. et al. Parasitization of vascular bundles of Anthurium rhizomes by Radopholus similis. Nematropica, v.33, p.209-213, 2003. Disponível em: <http://journals.fcla.edu/ nematropica/article/view/69677/67337>. Acesso em: 08 nov. 2012.

ZEM, A.C.; LORDELLO, L.G.E. Estudos sobre hospedeiros de Radopholus similis e Helicotylenchus multicinctus. Sociedade Brasileira de Nematologia, v.7, p.175-188, 1983. Disponível em: $\quad<$ http://docentes.esalq.usp.br/sbn/nbonline/ol\%2007u/175187\%20pb.pdf>. Acesso em: 03 mar. 2012. 